\title{
Positron Annihilation in Polycrystalline Metals Deformed by Uniaxial Tension
}

\author{
W. Rudzińska, J. Paja̧k, M. Szuszkiewicz, G. Bujnarowski \\ AND A.A. KLUZA \\ Institute of Physics, Opole University, Oleska 48, 45-052 Opole, Poland
}

\begin{abstract}
Angular distributions of the positron annihilation quanta were measured for polycrystalline samples deformed by uniaxial tension up to different deformation degrees. The $S$ parameter as a function of the $W$ parameter was determined. The data obtained for samples elongated up to different elongation degrees indicate that in the proportionality and limited proportionality regions the changes in the physical properties of samples are governed mainly by generation of vacancies and formation and kinetics of transformations of vacancy clusters occurring first of all on the grains of monocrystallites. In the region of plastic deformations the dominant defects are dislocations and vacancies and their aggregates generated due to the formation and movement of the dislocation of the primary and secondary slip. The positron annihilation data are corroborated by the results of the resistometric and tensometric measurements in the proportionality and limited proportionality regions.
\end{abstract}

PACS numbers: $78.70 . \mathrm{Bj}$

\section{Introduction}

The static uniaxial tensile test is the simplest method of investigation of mechanical properties of materials. Its result is the dependence of the relative deformation $\varepsilon$ on the value of applied static load. Analysis of this dependence permits to define the ranges of the elastic, elasto-plastic and plastic behavior of investigated material. Figure 1 represents a typical static strain-stress curve with all the possible ranges of deformation which may occur in the static uniaxial tensile tests.

From the physical point of view interesting is the picture of the processes occurring in particular deformation intervals, especially in polycrystalline samples. The interpretation of deformation characteristics is, however, difficult due to the 


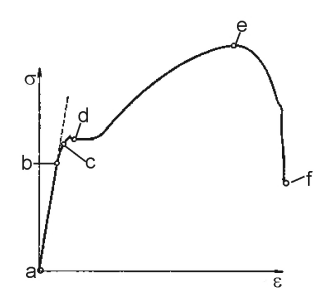

Fig. 1. Engineering (real) strain-stress curve: $a-b-$ elastic range, $b-c-$ limited elasticity range, $c-d-$ plasticity range, $d-e-$ plastic range, $e-f-$ flow of the sample, $f$ - rupture.

complexity of dislocation processes occurring both in the grains (monocrystallites) as well as on the grain boundaries in polycrystalline materials.

It is well known that polycrystalline material is an ensemble of microcrystallites with randomly oriented crystal axes (Fig. 2). They have no defined elasticity limit [1].

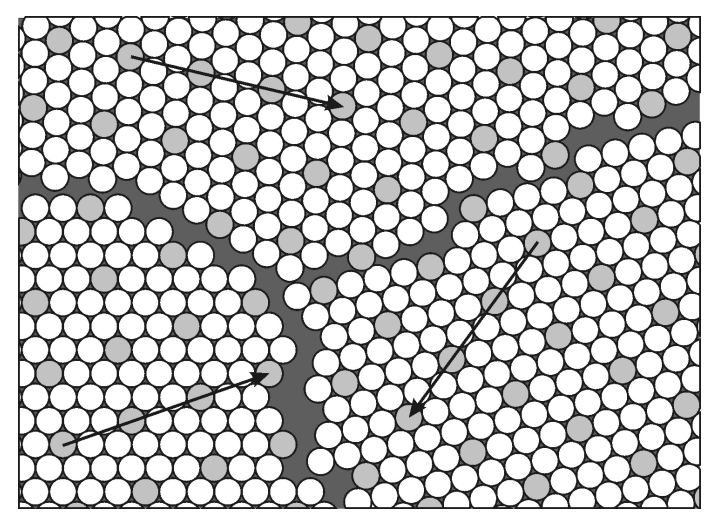

Fig. 2. Sketch of the polycrystalline structure. The black areas represent the intergrain regions. The randomly oriented arrows denote some crystalline direction, the same in each of the grains (monocrystallites). The atoms occupy equivalent sites located along the same crystalline direction in each of the grains.

Even in the equilibrium state, the grain boundaries are strongly defected. After exceeding some crystal strain the defects accumulated at the grain boundaries penetrate through the grain boundaries. In the macroscopic scale this results in appearance of the low and upper limits of elasticity. In these intervals the strain oscillates around some value proportional to the energy required for penetration of the grain boundary by the defect. In this interval some ordering of the intergrain regions is observed.

After equalization of the concentration of dislocations in all the grains, their penetration through the grain boundaries becomes unimportant. At this moment 
the plasticity arrest $(c-d)$ ends and the plastic range $(d-e)$ starts. The dislocations (especially the edge dislocations) strongly interact among themselves and the hardening of the sample starts. Although the cross-section area of the sample decreases uniformly along the sample length, the strain increases rapidly because of the hardening of the sample. In this range the mutual displacements of the grains, their coalescence (because of joining of small grains and decomposition of great grains into small pieces) is observed.

In the case of polycrystalline materials the flow range $(e-f)$ appears faster than in the case of monocrystals, because the volume of the intergrain regions is much greater than the volume of such regions generated in the tensile test of monocrystals. Independent of the initial microstructure, similarly as in monocrystals, the microcracks appear, especially at the broad angle grain boundaries which are the regions of smaller strength. The appearance of the microcracks leads to the sample rupture.

The present study is an attempt of observation of the microscopic structural changes occurring during the static tensile tests of the Ta, Fe, and Ag samples basing on the changes of the positron annihilation parameters measured during the test.

\section{Experimental techniques}

Before the experiments samples of $99.5 \%$ purity metals were initially annealed in vacuum (the pressure lower than $10^{-5}$ mbar) during $1 \mathrm{~h}$ at temperature $1123 \mathrm{~K}$ and then slowly cooled down $(\approx 1 \mathrm{~K} / \mathrm{min})$ to room temperature.

Samples of polycrystals with dimensions $1 \times 8 \times 30 \mathrm{~mm}^{3}$ were used for investigations of the influence of external stretching. The angular correlation curves of positron annihilation quanta (RKKA) were determined by means of a standard scintillation spectrometer described in detail in [2]. The positron source ${ }^{22} \mathrm{Na}$ of the activity of about $10 \mathrm{mCi}$ was used. The resistivity and tensometric measurements were conducted by means of the methods described in [3]. All the measurements were performed at room temperature under normal pressure.

\section{Results and discussion}

In order to bring into evidence the common features of the processes occurring during the static tensile test, the dependences of the annihilation parameter $S$ on the relative elongation $\varepsilon$ of all the investigated metals ( $\mathrm{Ta}, \mathrm{Fe}, \mathrm{Ag}$ ) are shown together in Fig. 3. We analyse the differences in the $S$ parameter value with respect to that for unloaded sample, therefore the differences in the absolute values are unimportant. The broken line in the figures is an interpolation of the experimental points. A detailed analysis of the changes in the $S$ parameter value (Fig. 3) indicates a pronounced increase in the $S$ parameter in the initial stage of the sample 
loading, followed by a minimum after which the $S$ parameter value monotonically increases up to some saturation value. The changes in the $S$ parameter value for Fe and $\mathrm{Ag}$ are more pronounced than those for Ta. The initial increase in the $S$ parameter value can be ascribed to the range of the proportionality between the strain and elongation.

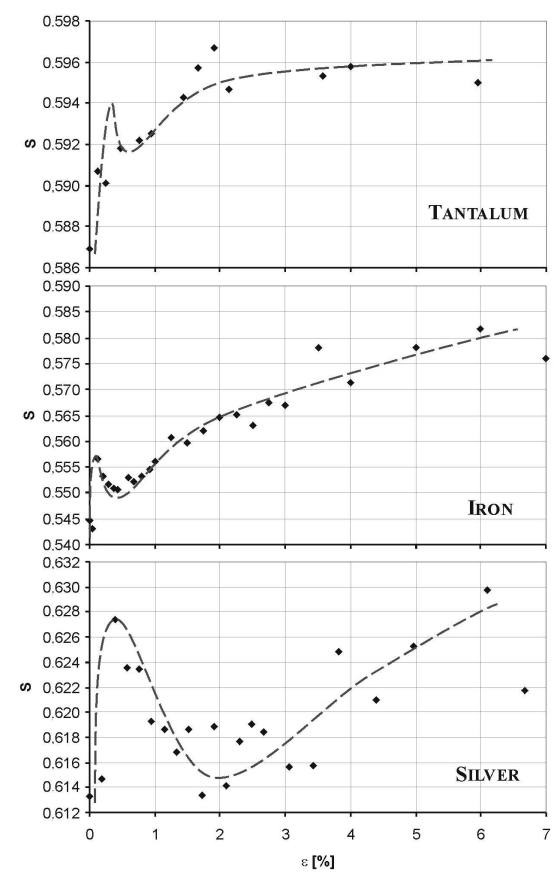

Fig. 3. Dependence of the $S$ parameter on the relative elongation $\varepsilon$ for tantalum, iron, and silver samples.

Taking into account the main assumptions of the Grifkinsal model [4], according to which the decoherence boundaries of individual microcrystals consist of linear configurations of vacancies in the decoherence planes, as well as the fact that the majority of microcrystals deforms elastically, it is reasonable to assume that the observed increase in the $S$ parameter value is caused by appearance of new positron trapping defects like vacancies and their agglomerations. With further loading of the samples the concentration of nonequilibrium vacancies decreases because of the activation of dislocations and movement of the crystalline planes in microcrystallites along the easy slip planes. Therefore the $S$ parameter decreases up to some minimum value.

It is well known that the share of dislocations in the changes of the $S$ parameter value is much smaller than that of vacancies. Therefore, further loading of the sample increasing the concentration of dislocations does not compensate the effect of decreasing vacancy concentration. Location of the minimum value of 
the $S$ parameter can be ascribed to the region of the maximum mobility of dislocations - the elasto-plastic range in the strain-stress curve. Further increase in the sample load causes further increase in the concentration of dislocations, and their blocking both in the volume of grains and in their boundaries. Therefore the mobility of dislocations decreases and simultaneously the internal stress increases. This in turn causes an increase in the vacancy concentration resulting in an observed increase in the $S$ parameter value up to some saturation level (Fig. 3 ).

From the interpolated $S-\varepsilon$ curves (broken lines in Fig. 3) the coordinates of the extrema $\left(S_{\max }, \varepsilon_{\max }\right),\left(S_{\min }, \varepsilon_{\min }\right)$ were determined and collected in Table. An inspection of the data collected in Table shows that maximum value of the $S$ parameter for iron occurs at the smallest and that for silver at the greatest value of the relative elongation $\varepsilon$. The sequence of the location of $S$ maxima correlate well with the sequence of the values of the Young modulus (Fe - $200 \mathrm{GPa}$, Ag - $190 \mathrm{GPa}$, Ta - $74 \mathrm{GPa}$ [5]). This correlation, together with the commonly accepted assumption that the stress corresponding to the lower plasticity limit of metals does not exceed $0.5 \varepsilon$, suggests that the location of the $S$ parameter maxima corresponds to the upper limit for elasto-plastic deformation of investigated materials.

TABLE

Values of the parameters determined from Fig. 3.

\begin{tabular}{c|c|c|c}
\hline \hline $\begin{array}{c}\text { Sample } \\
\text { parameters }\end{array}$ & Tantalum & Iron & Silver \\
\hline$\varepsilon_{\max }$ & 0.28 & 0.13 & 0.38 \\
$S_{\max }$ & 0.594 & 0.558 & 0.628 \\
$\varepsilon_{\min }$ & 0.63 & 0.44 & 1.88 \\
$S_{\min }$ & 0.592 & 0.550 & 0.615
\end{tabular}

Location of the $S_{\min }$ value could be connected with the plasticity arrest $(c-d$ range in Fig. 1). We assume that the measure of the plasticity arrest is the halfwidth of the broken curve in Fig. 3 fitting the experimental points in the region of maximum $S$ value. It should be noted that for silver the plasticity arrest is the largest. The plasticity arrests for tantalum and iron are comparable, but distinctly shorter than that for silver. This reflects, very probably, the difference in the crystal structure of investigated materials ( $A 1$ for silver and $A 2$ for Ta and $\mathrm{Fe})$.

For iron and silver the dependences of the $S$ parameter value on the relative elongation correspond well with the dependences of the resistivity of these materials on their relative elongation reported in [6]. Figure 4 represents the correlations between the $S$ and $W$ parameter values determined for each of the relative elon- 
gation values. Two straight lines are used to fit the experimental points for each of investigated materials. One of these straight lines fits the points corresponding to the interval $0<\varepsilon<\varepsilon_{\min }$. The remaining points lie in the close vicinity of the second straight line. The two straight lines form some angle. It is well known from the literature that the $\Delta S / \Delta W$ ratio is constant if the positrons are trapped only in one type of defects. The value of $\Delta S / \Delta W$ ratio, named $R_{\mathrm{D}}$ parameter, determines the dimensions of the defect. A detailed description of this conjecture may be found in [7-9]. In such a case the experimental points on the $S-W$ curve are located along one straight line. When the positrons are trapped in defects of different types (vacancies, dislocations, grain boundaries) the $R_{\mathrm{D}}$ parameter value gives the dimension of defects, averaged over all the positron trapping centers present in the investigated sample.
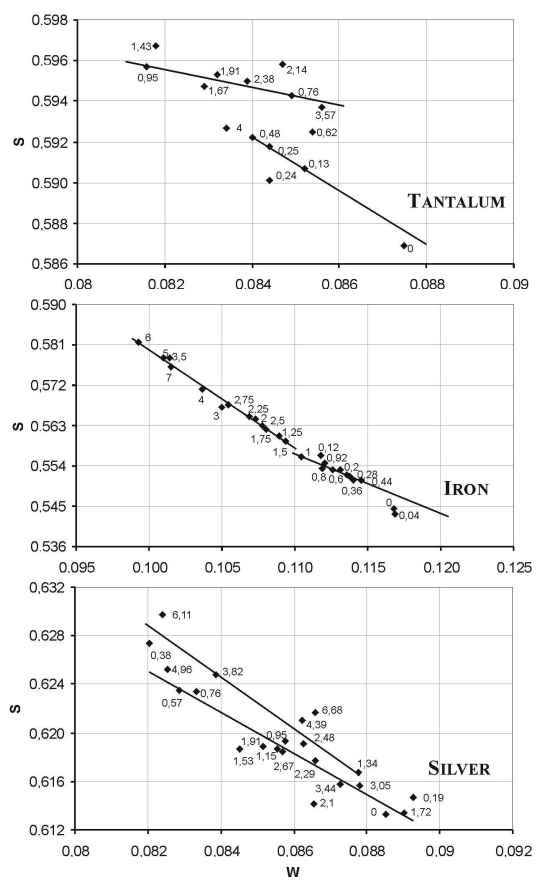

Fig. 4. Correlation between the $S$ parameter values for three investigated metals (Ta, $\mathrm{Fe}, \mathrm{Ag})$.

The value of the $R_{\mathrm{D}}$ parameter may change in the course of experiment. In this case the $S-W$ curve must be fitted by more than one straight line.

As it follows from Fig. 4, in different ranges of uniaxial tension different types of defects play a dominant role in positron trapping in investigated materials. In the range of elasto-plastic deformation the vacancies and their aggregates generated mainly in the grain boundary regions play the dominant role. In the remaining ranges of the strain-stress curve the dominant role belongs to the va- 
cancy type defects formed due to the generation and movement of dislocations in the primary secondary slip.

\section{Conclusions}

From the investigations performed in the frames of the present study it follows out that:

- measurements of the angular correlations of positron annihilation quanta can be applied as a method supporting the static tensile tests;

- changes in the positron annihilation parameters caused by uniaxial static elongation permit to reveal and to describe the microscopic changes in the structure of investigated material;

- it is possible to follow the kinetics of generation of defects with conjecture to the particular ranges of the strain-stress curve;

- the course of the dependence of the $S$ parameter value on the relative elongation curve corresponds well with the shape of the real strain-stress curve;

- it is worthy performing the complimentary investigations of the changes in the positron lifetime and in the electrical resistivity of investigated materials in the static tensile tests. Such measurements are in the final stage of realization and their results will be published in the nearest future [6].

\section{References}

[1] F. Staub, J. Adamczyk, Ł. Cieślak, J. Gubała, A. Maciejny, Metallography, Ślạsk, Katowice 1979 (in Polish).

[2] Cz. Szymański, S. Chabik, J. Pająk, B. Rozenfeld, Phys. Status Solidi A 60, 375 (1980).

[3] R. Pietrzak, R. Szatanik, M. Szuszkiewicz, J. Alloys Comp. 282, 130 (1999).

[4] M. Głowacka, Metallography, Wyd. Politechnika Gdańska, Gdańsk 1996 (in Polish).

[5] T. Szymczyk, S. Rabiej, A. Pielesz, J. Desselberger: Mathematical, Physical, Chemical and Astronomical Data, Cykada, Warszawa 2002 (in Polish).

[6] W. Rudzińska, J. Pająk, M. Szuszkiewicz, G. Bujnarowski, A.A. Kluza, Phys. Scr. 71, 111 (2005).

[7] St. Chabik, W. Rudzińska, Cz. Szymański, M. Szuszkiewicz, in: Proc. 33rd Polish Seminar on Positron Annihilation, Turawa (Poland) 2001, Ed. K. Jerie, Institute of Physics, University of Opole, Institute of Experimental Physics, University of Wrocław, Opole 2001, p. 73.

[8] W. Triftshäuser, Phys. Rev. B 12, 4634 (1975).

[9] J. Dryzek, Introduction to the Spectroscopy of Positron Annihilation in Condensed Matter, Jagiellonian University, Kraków 1999 (in Polish). 